\title{
Zusatzversicherungen steuerlich geltend machen?
}

- In einem vor dem baden-württembergischen Finanzgericht anhängigen Verfahren steht die Streitfrage zur Klärung, ob die im Einkommensteuergesetz (EStG) festgelegte Regelung des Sonderausgabenabzugs verfassungskonform ist. Nach dieser Vorschrift ist der Abzug von Sonderausgaben (d. h. insbesondere über das sozialhil- fegleiche Versorgungsniveau hinausgehende Kranken- und Pflege-, Unfall-, Haftpflicht- und Risikolebensversicherungen) nicht mehr möglich, wenn die Aufwendungen für sozialhilfegleiche Kranken- und Pflegeversicherungen, die abgezogen werden können, die im EStG vorgesehenen Höchstbeträge übersteigen.

\section{MMW Kommentar}

Durch die Neuregelungen des Bürgerentlastungsgesetzes können ab 2010 geleistete Beiträge zur (Basis-)Kranken- und Pflegeversicherung - auch bei Privatversicherung - in vollem Umfang als Sonderausgaben steuerlich geltend gemacht werden. Demgegenüber sind die Beiträge zu weiteren Versicherungen, die nicht zur Basisabsicherung dienen (wie z.B. Kranken- oder Krankenhaustagegeldversicherungen), nur beschränkt abzugsfähig. Hierfür wurde vom Gesetzgeber lediglich ein erhöhtes Abzugsvolumen in
Höhe von 1900 bzw. 2800 Euro (Ledige/Verheiratete) eingeführt.

Mit Urteil vom 16.11.2011 hat der Bundesfinanzhof diese Regelung des Gesetzgebers zwar bestätigt, gegen diese Entscheidung ist jedoch Verfassungsbeschwerde beim Bundesverfassungsgericht (BVG) eingelegt worden (Aktenzeichen 2 BvR 598/12). Betroffene Steuerpflichtige können deshalb Einspruch gegen ihren Steuerbescheid einlegen und das Ruhen des Verfahrens bis zu einer Entscheidung des BVG beantragen.

\section{Vorsicht Praxisgebühr!}

- Die Praxisgebühr soll abgeschafft werden. Bis zu diesem Zeitpunkt bleibt sie aber ein Dauerthema in jeder Praxis. Besonderer Beachtung bedürfen dabei jene Fälle, bei denen vom Patienten eine Befreiung von der Zuzahlung reklamiert wird. Solche Befreiungsausweise sollte man genau prüfen und insbesondere auf den Gültigkeitszeitraum achten. Zu Beginn eines Jahres werden u. U. Befreiungen des Vorjahres vorgelegt, und gefälschte Befreiungsausweise sind mittlerweile auch schon aufgetaucht.

\section{MMW Kommentar}

Es lohnt sich auf jeden Fall, die ausgewiesenen Beträge über die eingenommenen Praxisgebühren im Abrechnungsbescheid mit den Daten aus dem Praxisverwaltungssystem zu vergleichen. Differenzen sollten der zuständigen KV gemeldet und um Klärung des Sachverhaltes gebeten werden.

\section{Neuregelungen bei der Laborabrechnung}

- Mit Einführung des EBM 2000plus wurde für den hausärztlichen Bereich geregelt, dass die Nrn. 01730 und 01735 EBM (bezogen auf Kapitel 3 des EBM) sowie die Nrn. 01816, 01821, 01822, 01828, 01840 und 01915 EBM von den unter Nummer $1 \mathrm{im}$ Kapitel 3 und 4 genannten Vertragsärzten nur dann berechnungsfähig sind, wenn sie eine mindestens einjährige Weiterbildung im Gebiet Frauenheilkunde und Geburtshilfe nachweisen können oder wenn entsprechende Leistungen bereits vor dem 31. Dezember 2002 durchgeführt und abgerechnet wurden. Unter der Nr. 01828 EBM kann seit dem 1. Juli 2012 nur die Ent-

nahme von Venenblut für den Varizella-Zoster-Virus-Antikörpernachweis im Rahmen der Empfängnisregelung abgerechnet

\section{MMW Kommentar}

Die Schutzimpfungs-Richtlinie führt gemäß Empfehlungen der ständigen Impfkommission (STIKO) aus, dass bei Frauen mit dokumentierter zweimaliger Rötelnimpfung von Immunität auszugehen ist und eine Antikörperbestimmung in diesen Fällen nicht erforderlich ist. Lediglich Frauen mit fehlender oder nur einmaliger Impfung soll die Rötelnimpfung beziehungsweise deren werden. Die Entnahme von Venenblut für den Röteln-HAH-Test ist nicht mehr berechnungsfähig.
Komplettierung empfohlen werden. Die serologische Überprüfung der Immunitätslage ist nicht mehr vorgesehen. Entsprechend wurde die Nr.01829 EBM „Rötelnantikörper im Hämagglutinationshemmungstest ( $\mathrm{HAH}$ ) bei ungeklärter Immunitätslage gegen Röteln im Rahmen der Empfängnisregelung" gestrichen. 